\title{
Lecture Notes on Mathematical Olympiad Courses
}

For Senior Section Vol. 2 


\section{Mathematical Olympiad Series}

ISSN: 1793-8570

Series Editors: Lee Peng Yee (Nanyang Technological University, Singapore)

Xiong Bin (East China Normal University, China)

\section{Published}

Vol. 1 A First Step to Mathematical Olympiad Problems by Derek Holton (University of Otago, New Zealand)

Vol. 2 Problems of Number Theory in Mathematical Competitions by Yu Hong-Bing (Suzhou University, China) translated by Lin Lei (East China Normal University, China)

Vol. 3 Graph Theory

by Xiong Bin (East China Normal University, China) \&

Zheng Zhongyi (High School Attached to Fudan University, China) translated by Liu Ruifang, Zhai Mingqing \& Lin Yuanqing

(East China Normal University, China)

Vol. 4 Combinatorial Problems in Mathematical Competitions by Yao Zhang (Hunan Normal University, P. R. China)

Vol. 5 Selected Problems of the Vietnamese Olympiad (1962-2009) by Le Hai Chau (Ministry of Education and Training, Vietnam) \& Le Hai Khoi (Nanyang Technology University, Singapore)

Vol. 6 Lecture Notes on Mathematical Olympiad Courses: For Junior Section (In 2 Volumes) by Xu Jiagu

Vol. 7 A Second Step to Mathematical Olympiad Problems by Derek Holton (University of Otago, New Zealand \& University of Melbourne, Australia)

Vol. 8 Lecture Notes on Mathematical Olympiad Courses: For Senior Section (In 2 Volumes) by Xu Jiagu 


\section{$\mathrm{Xu}$ Jiagu}

Former Professor of Mathematics, Fudan University, China

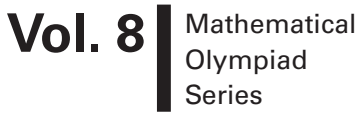

\section{Lecture Notes on Mathematical Olympiad Courses}

For Senior Section Vol. 2 


\section{Published by}

World Scientific Publishing Co. Pte. Ltd.

5 Toh Tuck Link, Singapore 596224

USA office: 27 Warren Street, Suite 401-402, Hackensack, NJ 07601

UK office: 57 Shelton Street, Covent Garden, London WC2H 9HE

\section{British Library Cataloguing-in-Publication Data}

A catalogue record for this book is available from the British Library.

\section{Mathematical Olympiad Series - Vol. 8 \\ LECTURE NOTES ON MATHEMATICAL OLYMPIAD COURSES \\ For Senior Section \\ (In 2 Volumes)}

Copyright () 2012 by World Scientific Publishing Co. Pte. Ltd.

All rights reserved. This book, or parts thereof, may not be reproduced in any form or by any means, electronic or mechanical, including photocopying, recording or any information storage and retrieval system now known or to be invented, without written permission from the Publisher.

For photocopying of material in this volume, please pay a copying fee through the Copyright Clearance Center, Inc., 222 Rosewood Drive, Danvers, MA 01923, USA. In this case permission to photocopy is not required from the publisher.

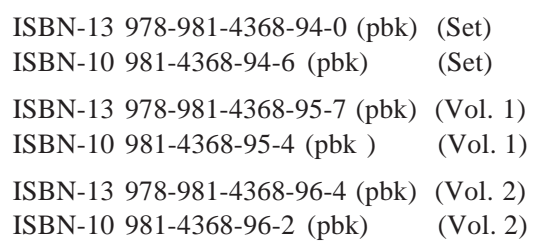

Printed in Singapore. 


\title{
Preface
}

\begin{abstract}
Although Mathematical Olympiad competitions are carried out by solving problems, the system of Mathematical Olympiads and the related training courses cannot consist only of problem solving techniques. Strictly speaking, it is a system of mathematical advancing education. To guide students, who are interested in and have the potential to enter the world of Olympiad mathematics, so that their mathematical ability can be promoted efficiently and comprehensively, it is important to improve their mathematical thinking and technical ability in solving mathematical problems.
\end{abstract}

An excellent student should be able to think flexibly and rigorously. Here, the ability to perform formal logic reasoning is an important basic component. However, it is not the main one. Mathematical thinking also includes other key aspects, such as starting from intuition and entering the essence of the subject, through the processes of prediction, induction, imagination, construction and design to conduct their creative activities. In addition, the ability to convert the concrete to the abstract and vice versa is essential.

Technical ability in solving mathematical problems does not only involve producing accurate and skilled-computations and proofs using the standard methods available, but also the more unconventional, creative techniques.

It is clear that the standard syllabus in mathematical education cannot satisfy the above requirements. Hence the Mathematical Olympiad training books must be self-contained basically.

This book is based on the lecture notes used by the editor in the last 15 years for Olympiad training courses in several schools in Singapore, such as Victoria Junior College, Hwa Chong Institution, Nanyang Girls High School and Dunman High School. Its scope and depth significantly exceeds that of the standard syllabus provided in schools, and introduces many concepts and methods from modern mathematics. 
The core of each lecture are the concepts, theories and methods of solving mathematical problems. Examples are then used to explain and enrich the lectures, as well as to indicate the applications of these concepts and methods. A number of questions are included at the end of each lecture for the reader to try. Detailed solutions are provided at the end of book.

The examples given are not very complicated so that the readers can understand them easily. However, many of the practice questions at the end of lectures are taken from actual competitions, which students can use to test themselves. These questions are taken from a range of countries, such as China, Russia, the United States of America and Singapore. In particular, there are many questions from China for those who wish to better understand Mathematical Olympiads there. The questions at the end of each lecture are divided into two parts. Those in Part A are for students to practise, while those in Part B test students' ability to apply their knowledge in solving real competition questions.

Each volume can be used for training courses of several weeks with a few hours per week. The test questions are not considered part of the lectures as students can complete them on their own. 


\section{Acknowledgments}

My thanks to Professor Lee Peng Yee for suggesting the publication of this the book, and to Professor Phua Kok Khoo for his strong support. I would also like to thank my former excellent student, Mr Tay Jingyi Kenneth, for his corrections, as well as Zhang Ji and He Yue, the editors of this book at World Scientific Publishing Co. (WSPC). This book would not have been published without their efficient assistance. 
This page intentionally left blank 


\section{Abbreviations and Notations}

\section{Abbreviations}

AHSME American High School Mathematics Examination

AIME American Invitational Mathematics Examination

AMC American Mathematics Examination

APMO Asia Pacific Mathematics Olympiad

ASUMO Olympics Mathematical Competitions of All the Soviet Union

AUSTRIA Austria Mathematical Olympiad

AUSTRALIA Australia Mathematical Olympiad

BALKAN Balkan Mathematical Olympiad

BELARUS Belarus Mathematical Olympiad

BMO British Mathematical Olympiad

BOSNIA Bosnia Mathematical Olympiad

BULGARIA Bulgaria Mathematical Olympiad

CGMO China Girl's Mathematical Olympiad

CHINA China Mathematical Competition for Secondary Schools except for CHNMOL

CHNMOL China Mathematical Competition for Secondary Schools

CHNMO China Mathematical Olympiad

CMC China Mathematical Competition (for High Schools)

CMO Canada Mathematical Olympiad

CNMO China Northern Mathematical Olympiad

CSMO China Southeastern Mathematical Olympiad

CROATIA Croatia Mathematical Olympiad

CWMO China Western Mathematical Olympiad 
CZECH-POLISH-SLOVAK International Competitions Czech-Polish-Slovak Match

ESTONIA Estonia Mathematical Olympiad

GERMANY Germany Mathematical Olympiad

GREECE Greece Mathematical Olympiad

High-School Mathematics Journal of "High-School Mathematics", China.

HONG KONG Hong Kong Mathematical Olympiad

HUNGARY Hungary Mathematical Competition

HUNGARY-ISRAEL Hungary-Israel Binational Mathematical Competition

IMO International Mathematical Olympiad

INDIA India Mathematical Olympiad

IRAN Iran Mathematical Olympiad

IRE Ireland Mathematical Olympiad

JAPAN Japan Mathematical Olympiad

KOREA Korea Mathematical Olympiad

MACEDONIA Macedonia Mathematical Olympiad

MEDITERRANEAN MO Mediterranean Mathematics Olympiad

POLAND Poland Mathematical Olympiad

PUTNAM Putnam Mathematical Competition

ROMANIA Romania Mathematical Olympiad

RUSMO All-Russia Olympics Mathematical Competitions

SAUDI ARABIA Saudi Arabia Mathematical Olympiad

SERBIA Serbia Mathematical Olympiad

SLOVENIA Slovenia Mathematical Olympiad

SMO Singapore Mathematical Olympiads

SSSMO Singapore Secondary Schools Mathematical Olympiads

SWE Sweden Mathematical Olympiads

TAIWAN Taiwan Mathematical Olympiads

THAILAND Thailand Mathematical Olympiads

TST Team Selection Test (including related training tests)

TURKEY Turkey Mathematical Olympiad

UKRAINE Ukraine Mathematical Olympiad

USAMO United States of America Mathematical Olympiad

USSR Union of Soviet Socialist Republics

VIETNAM Vietnam Mathematical Olympiad 


\title{
Notations for Numbers, Sets and Logic Relations
}

\author{
$\mathbb{N}$ the set of positive integers (natural numbers) \\ $\mathbb{N}_{0}$ the set of non-negative integers \\ $\mathbb{Z}$ the set of integers \\ $\mathbb{Z}^{+}$the set of positive integers \\ $\mathbb{Q}$ the set of rational numbers \\ $\mathbb{Q}^{+}$the set of positive rational numbers \\ $\mathbb{Q}_{0}^{+} \quad$ the set of non-negative rational numbers \\ $\mathbb{R}$ the set of real numbers \\ $[m, n]$ the lowest common multiple of the integers $m$ and $n$ \\ $(m, n)$ the greatest common divisor of the integers $m$ and $n$ \\ $a \mid b \quad a$ divides $b$ \\ $|x|$ the absolute value of $x$ \\ $\lfloor x\rfloor$ the greatest integer not greater than $x$ \\ $\lceil x\rceil$ the smallest integer not less than $x$ \\ $\{x\}$ the decimal part of $x$, i.e. $\{x\}=x-\lfloor x\rfloor$ \\ $a \equiv b(\bmod c) \quad a$ is congruent to $b$ modulo $c$ \\ $\left(\begin{array}{l}n \\ k\end{array}\right) \quad$ the binomial coefficient $n$ choose $k$ \\ $n$ ! $n$ factorial, equal to the product $1 \cdot 2 \cdot 3 \cdots n$ \\ $[a, b]$ the closed interval, i.e. all $x$ such that $a \leq x \leq b$ \\ $(a, b)$ the open interval, i.e. all $x$ such that $a<x<b$ \\ $\Leftrightarrow \quad$ iff, i.e. if and only if \\ $\Rightarrow$ implies \\ $A \subset B \quad A$ is a subset of $B$ \\ $A-B$ the set formed by all the elements in $A$ but not in $B$ \\ $A \cup B$ the union of the sets $A$ and $B$ \\ $A \cap B \quad$ the intersection of the sets $A$ and $B$ \\ $a \in A \quad$ the element $a$ belongs to the set $A$
}


This page intentionally left blank 


\section{Contents}

Preface $\quad$ v

Acknowledgments vii

$\begin{array}{ll}\text { Abbreviations and Notations } & \text { ix }\end{array}$

16 Mathematical Induction 1

17 Arithmetic Progressions and Geometric Progressions 11

18 Recursive Sequences $\quad 19$

19 Summation of Various Sequences 29

20 Some Fundamental Theorems on Congruence $\quad 37$

21 Chinese Remainder Theorem and Order of Integer 45

22 Diophantine Equations (III) 53

23 Pythagorean Triples and Pell's Equations 61

24 Quadratic Residues

25 Some Important Inequalities (I) 75

26 Some Important Inequalities (II) 85

27 Some Methods For Solving Inequalities 93

28 Some Basic Methods in Counting (I) 101

29 Some Basic Methods in Counting (II) 109 
30 Introduction to Functional Equations

Solutions to Testing Questions

Appendices

A Theorem on Second Order Recursive Sequences 261

B Proofs of Theorems On Pell's Equation 263

C Theorems On Quadratic Residues 267

D Proofs of Some Important Inequalities 273

E Note On Cauchy's Problem in Functional Equations 279

$\begin{array}{ll}\text { Index } & 281\end{array}$ 